\title{
Error and Attack Tolerance of Layered Complex Networks
}

\author{
Maciej Kurant: and Patrick Thiran \\ EPFL, Switzerland
}

(Dated: October 30, 2018)

\begin{abstract}
Many complex systems may be described not by one, but by a number of complex networks mapped one on the other in a multilayer structure 11. The interactions and dependencies between these layers cause that what is true for a distinct single layer does not necessarily reflect well the state of the entire system. In this paper we study the robustness of three real-life examples of twolayer complex systems that come from the fields of communication (the Internet), transportation (the European railway system) and biology (the human brain). In order to cover the whole range of features specific to these systems, we focus on two extreme policies of system's response to failures, no rerouting and full rerouting. Our main finding is that multilayer systems are much more vulnerable to errors and intentional attacks than they seem to be from a single layer perspective.
\end{abstract}

PACS numbers: 89.75.Hc, 89.75.Fb, 89.40.Bb, 89.20.Hh

The robustness of a complex system can be defined by how it behaves under stress. There are two general categories of such stress: errors - failures of random components, and attacks - failures of components that play a vital role in the system. Recently, many complex systems have been successfully described in terms of complex networks 2 . These graphs may greatly differ in their response to failures. For instance, the 'scale-free' networks (i.e., networks whose node degree distribution is heavytailed [3]) such as World Wide Web, Internet, protein networks, ecological networks or cellular networks, exhibit remarkable robustness to errors, but at the same time, they are very vulnerable to attacks such as the removal of the most highly connected nodes [4] 5 5 [6] 7]. Subsequent studies of other attack strategies 8| 9], cascading failures 10] 11], defensive strategies 10| 12 13] 14] 15], and vulnerability of weighted networks 16 gave us valuable insights into the robustness of complex networks treated as distinct objects. Many of such networks, however, are only a part of larger systems, where a number of coexisting topologies interact and depend on each other [1]. For instance, in the Internet, a graph formed by an application (such as WWW or Peer-To-Peer) is mapped onto the IP network that, in turn, is mapped on a physical mesh of cables and optical fibers. The topology at every layer is different. Similarly, it is convenient to view a transportation network as a two-layer system, with a network of traffic demands mapped onto the physical infrastructure. This layered view sheds a new light on the issue of the error and attack tolerance of many complex systems. We show in this paper that what is observed at a single layer does not necessarily reflect well the state of the entire system. On the contrary - a tiny, seemingly unharmful (from one-layer perspective) disruption of the lower layer graph may destroy a substantial part of the upper layer graph rendering the whole system useless in practice.

*Electronic address: Maciej.Kurant@epfl.ch

\begin{tabular}{|l|r|r|r|r|r|r|}
\hline Data set & $\left|V^{\phi}\right|$ & $\left|E^{\phi}\right|$ & $\langle l\rangle$ & $\left|V^{\lambda}\right|$ & $\left|E^{\lambda}\right|$ & $\langle m\rangle$ \\
\hline Railway & $4^{\prime} 853$ & $5^{\prime} 765$ & 53.8 & $2^{\prime} 509$ & $7^{\prime} 038$ & 9.9 \\
Gnutella & $16^{\prime} 911$ & $37^{\prime} 849$ & 3.7 & $1^{\prime} 214$ & $31^{\prime} 193$ & 2.8 \\
Brain & $4^{\prime} 445$ & $20^{\prime} 967$ & 9.1 & $1^{\prime} 013$ & $15^{\prime} 369$ & 10.3 \\
\hline
\end{tabular}

TABLE I: Two-layer systems analyzed in this paper: 'Railway' - train traffic flows on top of the railway network of central Europe; 'Gnutella' - Gnutella P2P network on top of the AS level Internet; 'Brain' - long distance cortex-to-cortex axonal connections in the human brain on top of the 3D lattice in the white matter. $\langle l\rangle$ is the average shortest path length; $\langle m\rangle$ is the average mapping length.

A framework for an analysis of layered complex networks was recently introduced in [1]. In a two-layer case, the system consists of a weighted logical graph $G^{\lambda}=\left(V^{\lambda}, E^{\lambda}\right)$ and the underlying physical graph $G^{\phi}=$ $\left(V^{\phi}, E^{\phi}\right)$. The logical nodes are a subset of physical nodes, $V^{\lambda} \subset V^{\phi}$. Every logical edge $e^{\lambda}=\left(u^{\lambda}, v^{\lambda}\right)$ is mapped on the physical graph as a physical path $M\left(e^{\lambda}\right)$ connecting the nodes $u^{\phi}$ and $v^{\phi}$, corresponding to $u^{\lambda}$ and $v^{\lambda}$.

This layered framework allows us to study the robustness of the entire system. As the mapping of logical edges is usually longer than one hop, many physical links serve more than one logical edge (see Fig. 11). A failure of such a physical link affects all logical edges that are mapped on it. In other words, failures at the physical layer propagate to the logical layer, and at the same time they multiply. Moreover, the resulting failures at the logical layer are strongly correlated in time and space. These three phenomena make the response of a layered system to failures much more complex than what is observed at a single layer.

In our study we use three large examples of layered systems that come from fields as different as transportation, communication and biology. We present an overview of these data sets in Table $\square$ and describe each of them below.

Our first data set, called 'Railway', is the European railway system. It is extracted from timetables of $60 ' 775$ 


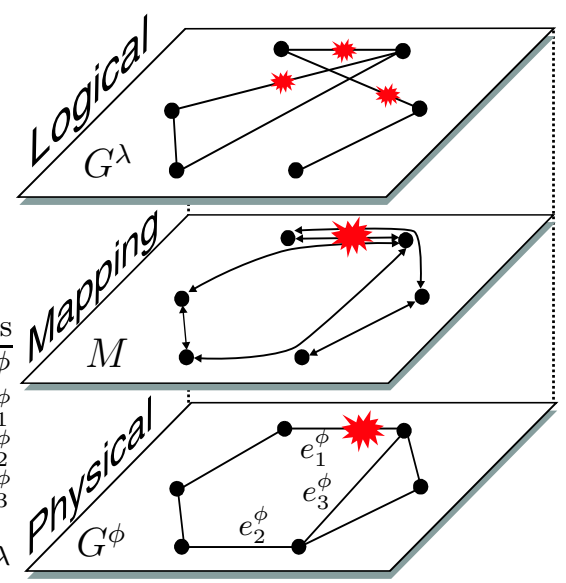

FIG. 1: Illustration of failure propagation, multiplication, and correlation in a two-layer system. A single failure in the physical graph results in three correlated failures in the logical graph.

trains in central Europe with the algorithm described in [17. The resulting physical graph reflects the reallife infrastructure that consists of 4'853 nodes (stations) and 5'765 edges (rail tracks). The logical graph contains 7'038 edges, each connecting the first and the last station of a train. The logical edge weight is the number of trains following the same route. The route itself is the mapping of this edge on the physical graph.

The second data set, called 'Gnutella', is an example of a large Peer-To-Peer (P2P) application in the Internet. In a P2P system the links between users are virtual and therefore they are usually created independently of the underlying Internet structure, forming a very different topology. Due to its immense size and dynamics, the exact map of the Internet at the IP level (i.e., where the nodes and IP routers and hosts) is still beyond our reach. Therefore we focus on its aggregated version, where each node is an Autonomous System AS (usually an Internet Service Provider), and where edges reflect the connections between the ASes. The topology of such AS-level Internet is well known thanks to numerous Internet mapping projects such as DIMES [18] or CAIDA 19]. For our physical graph we take the $09 / 2004$ topology provided by CAIDA, which consists of 16'911 nodes and 37'849 edges. For the logical graph we take a snapshot of the Gnutella P2P network collected in September 2004 by the crawler developed in [20]. It consists of around 1 million users, connected by several million links. In order to obtain the AS-level version of this network, we translated the IP addresses of the users into the corresponding AS numbers. All users with the same AS number become one node in the logical graph, and all links connecting the same pair of ASes become one edge of weight equal to the number of contributing links. As a result we obtain an AS-level logical graph of Gnutella with 1'214 nodes and 31'193 edges. The mapping of each logical edge is obtained by the shortest path in the physical graph connecting its end-nodes.

Our third data set, called 'Brain', captures the large scale connectivity of the human brain. It was inferred from MRI scans with the approach described in 21]. In particular, the brain cortex and the brain white matter are partitioned into a set of compact regions of comparable size. There are 1'013 regions in the cortex and 3'432 regions in the white matter. Every region becomes a node in the physical graph. The logical edges in this data set are the long distance axonal connections between the 1'013 regions in the cortex. Each such connection $e^{\lambda}$ traverses the white matter; the sequence of white matter regions on its path defines the mapping $M\left(e^{\lambda}\right)$. At the physical layer, two nodes are connected by a physical edge $e^{\phi}$ if they appear directly connected (i.e., are consecutive in the sequence of regions) in at least one mapping $M\left(e^{\lambda}\right)$. By this procedure we have obtained a two-layer structure, where the logical graph consists of the long-range connections in the brain and is mapped on the physical layer that reflects the ' $3 \mathrm{D}$ white matter structure' used to establish these long-range connections.

Of course, many real-life systems have mechanisms to partially or fully recover from failures. For instance, the Internet consists of several (seven layers in the classic view) layers that are specified in the ISO/OSI network model [22]. Some of these layers, e.g., the 'network layer' with its IP protocol, attempt to find an alternative path around a failing link or node. This requires, among others, the physical graph to be connected. The situation gets more difficult in railway networks, because for a train its entire path is important, not only the end-points. Although it is sometimes possible to slightly change the itinerary of the train or to organize alternative means of transportation (e.g., a bus) around the failing section, the common practice is to halt all the trains that use it. In order to keep our analysis general and to cover the whole spectrum of possible situations, in this paper we study two extreme policies: no rerouting, and full rerouting. In the former case we delete immediately all logical edges affected by a physical failure. In the latter case, we delete any affected logical edge $e^{\lambda}$ only when there is no path in the physical graph $G^{\phi}$ between the end-nodes of $e^{\lambda}$ (i.e., end-nodes of $e^{\lambda}$ belong to different components of $\left.G^{\phi}\right)$. Otherwise, the logical edge $e^{\lambda}$ remains in the graph, and its mapping is updated by the shortest path in $G^{\phi}$. Consider the example in Fig. 11 Under the no rerouting policy, three logical edges are removed after the failure of $e_{1}^{\phi}$. However, as the physical graph $G^{\phi}$ is still connected, under the full rerouting policy all these three logical links can be rerouted and thus remain in the logical graph.

By studying the two extreme policies, no rerouting and full rerouting, we also capture the specific features of our three data sets. For instance, in the railway system every rail track has a limited capacity that cannot be exceeded. Therefore, even if we allow for rerouting, some routes will be forbidden due to a possible overload. In the Gnutella data set, the AS graph routing depends on the internal 

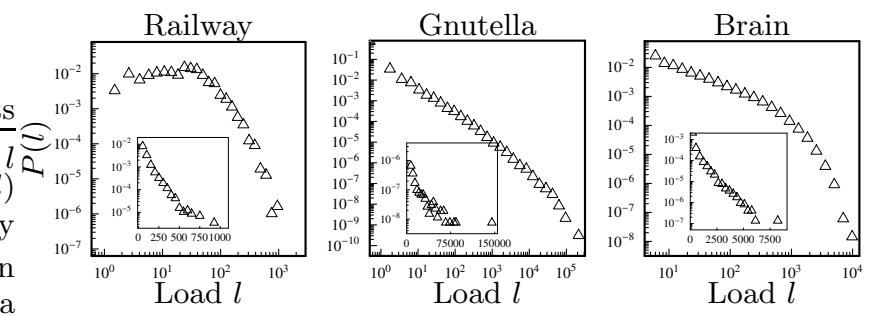

FIG. 2: Edge load distribution in three layered systems. The main plots are in log-log scale (log-binned); the insets present the same distributions in log-lin scale (lin-binned).

policy of involved ASes and peering relationships established between the ASes [23]. This results in routes that are not necessarily the shortest possible, and makes some of the routes invalid. These additional constraints imposed on the Railway and Gnutella paths naturally limit the performance of these systems below the 'full rerouting' level. Finally, the brain has some ability to reroute around broken connections too. However, this process takes substantial time. Therefore, an initial response of the brain would be better described by the no rerouting policy, but in time the brain will slowly recover and reroute some of the lost connections. This slow recovery process can be observed at patients that suffer from e.g., a stroke, or have undergone a brain surgery.

In other words, all responses of real systems to physical failures are located somewhere between the no rerouting and the full rerouting policy. This is especially important, because, as we show later the difference between these two extreme scenarios is often very small.

Before we simulate the impact of failures on our systems directly, let us try to roughly predict what will happen by studying related distributions. In a layered system, every physical node or edge can be characterized by the load. The load $l$ of the physical node $v^{\phi}$ or edge $e^{\phi}$ is the sum of weights of all the logical edges whose paths traverse $v^{\phi}\left(e^{\phi}\right)$ [1]. The load becomes a very important parameter when we allow for failures in the system. Clearly, the higher the load of a failing physical component, the more it affects and perturbs the logical layer. If the load is distributed evenly in the physical graph, a random failure will not be very different from an intentional attack. If, however, the load distribution is very uneven, the highly loaded parts become an obvious target for an efficient attack. In Fig. 2 2 we present the load distribution in the three layered systems we study. In each case the distribution is broad (covering 4-5 decades) and heavily right-skewed. This means that there is a significant number of physical links that carry a lot more traffic than the other links. Consequently, we can anticipate that an attack targeted on the most loaded links will harm the system much more efficiently than a random error scenario.

We verify this intuition by simulating the error and attack scenarios on the three studied systems. The results are presented in Fig. 3 Although the exact system response varies in all three cases, there are a number of features common to all or most of them:

1) The attacks are much more harmful than errors. For example, in Gnutella with no rerouting, half of the logical mass (total edge weight) is erased after $22 \%$ physical edges randomly fail, or after only $0.04 \%$ most loaded edges are attacked. Although under the 'full rerouting' policy this difference is smaller, we still need about 60 times more random failures than attacks to achieve the same goal.

2) When the system is attacked, the logical graph is usually affected much faster than the physical graph. For instance, in Gnutella, an attack (with or without rerouting) on $5 \%$ of the physical edges hardly affects the physical graph - the largest connected physical component covers almost the entire original graph. At the same time, this seemingly unharmful attack deletes more than $95 \%$ of logical edges! We obtain similar results when we consider the size of the largest connected component in the logical graph as the measure of robustness. (These results are not shown in Fig. 3 for better readability.)

3) The attack under the full rerouting policy affects the physical graph more than under no rerouting. When rerouting is allowed the logical edges are deleted only when the physical graph gets partitioned. This, in turn, effectively reduces the size of the largest connected physical component. This phenomenon is especially pronounced in the last plot in Fig. 3 (brain, attack tolerance). Under full rerouting, the size of the largest component in the physical graph (filled triangles) drops rapidly after about $55 \%$ of physical edges are attacked. Clearly, this component splits into two components of comparable size. This behavior can be explained on the example in Fig. 1 Initially, the physical edge $e_{1}^{\phi}$ is used by three logical links. It is the most loaded edge in the physical graph and hence it is removed as first by our attack. Now, under no rerouting policy, three logical edges are deleted. In what remains, the load is distributed equally on four physical edges, so there is no preferred edge for our attack. In particular, in the second round the attack may remove the physical edge $e_{3}^{\phi}$, keeping the physical graph connected. In contrast, under the full rerouting policy, after the removal of $e_{1}^{\phi}$ the three affected logical links are rerouted. As all of them must treverse the edge $e_{2}^{\phi}$, the load of $e_{2}^{\phi}$ increases to 4 and $e_{2}^{\phi}$ is removed in the second round of the attack. This efficiently splits the physical graph into two components of three nodes each. 25.

4) The logical graph is strongly affected by attacks regardless of the rerouting policy. This is expressed by the proximity of the filled and unfilled circles under attack in Fig. B (especially for Railway and Gnutella). As any real-life failure recovery policy falls between these two extremes (no rerouting and full rerouting), we expect this feature to be quite general and universal.

To conclude, the response of a multi-layer system to failures is much more complex than what is observed at a single layer. In particular, such systems are more vulner- 

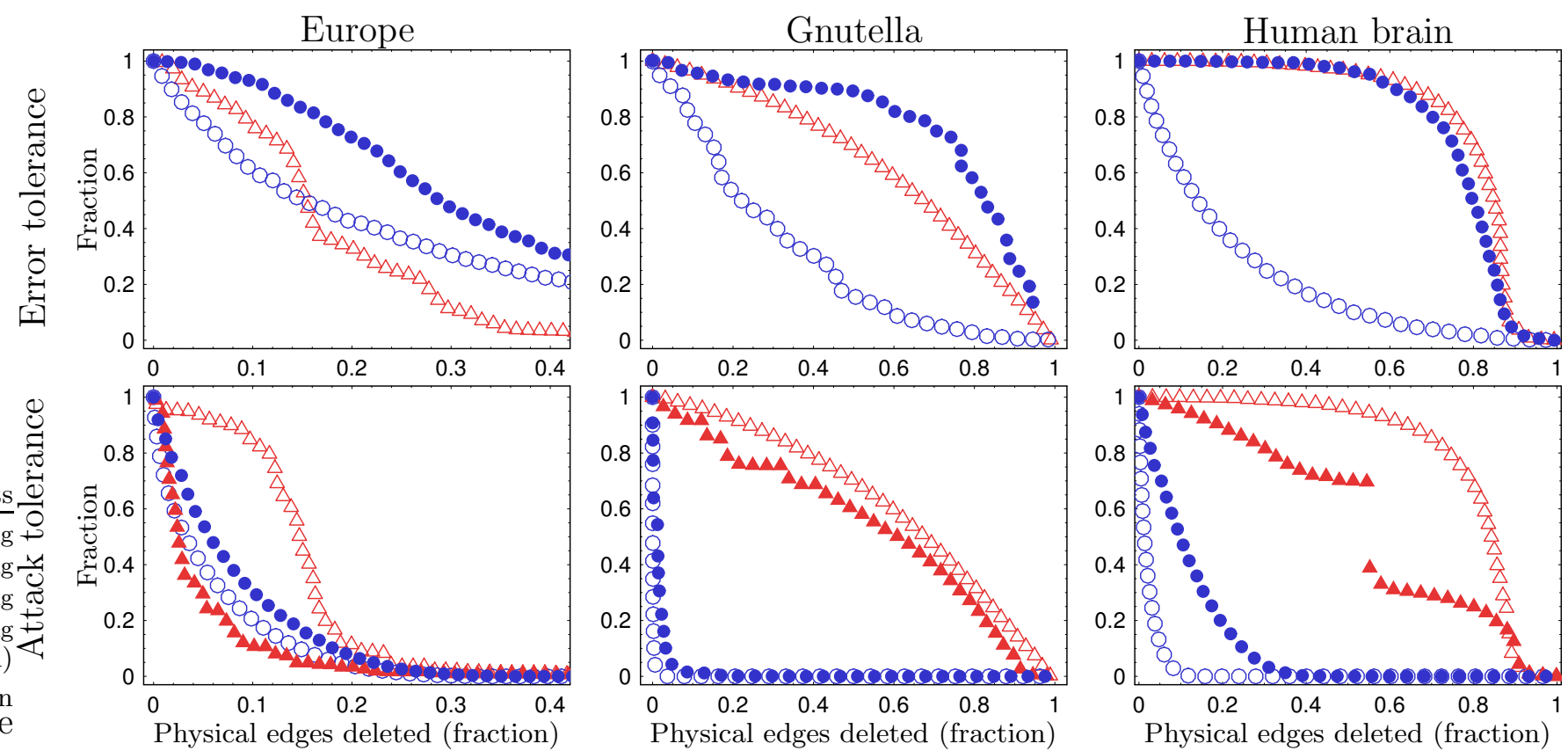

$\triangle$ Largest connected component in physical graph, no rerouting $\Delta$ Largest connected component in physical graph, full rerouting

Total weight of remaining logical edges, no rerouting

- Total weight of remaining logical edges, full rerouting

FIG. 3: Error and attack tolerance of three layered systems. At each iteration we remove one physical edge $e_{d e l}^{\phi}$ either at random ('error tolerance', top), or by choosing the most loaded one ('attack tolerance', bottom). In both cases we observe the size of the largest connected component in the physical graph $G^{\phi}$ (triangles) and the total weight of the remaining logical edges (circles). Every logical edge $e^{\lambda}$ whose mapping contains $e_{d e l}^{\phi}$ is deleted either directly ('no rerouting', unfilled symbols), or only when there is no path in $G^{\phi}$ between the end-nodes of $e^{\lambda}$ ('full rerouting', filled symbols).

able than they seem to be from a single layer perspective. This is very important, because the multi-layer structure is a model that fits well many real-life systems.

This work is only the first step towards understanding the behavior of layered systems under stress. There are numerous aspects that require further investigation. What is the impact of traffic locality, weight and load dis- tribution, failure correlation, or topological properties at the two layers on the robustness of the system? Do there exist attacks even more efficient than the one proposed in this paper? Is it possible to significantly improve the resilience of a system, e.g., by adding a relatively small number of physical or logical edges? We are planning to address these issues in our future work.
[1] M. Kurant and P. Thiran. Layered complex networks. Phys. Rev. Lett., 96(13):138701, April 2006.

[2] Mark Newman, Albert-Laszlo Barabasi, and Duncan J. Watts. The Structure and Dynamics of Networks. Princeton University Press, 2006.

[3] Barabási A. and Albert R. Emergence of scaling in random networks. Science, 286:509-512, 1999.

[4] R. Albert, H. Jeong, and A.-L. Barabási. Error and attack tolerance in complex networks. Nature, 406:378, 2000.

[5] Reuven Cohen, Keren Erez, Daniel ben Avraham, and Shlomo Havlin. Resilience of the internet to random breakdowns. Phys. Rev. Lett., 85:4626, 2000.

[6] Reuven Cohen, Keren Erez, Daniel ben Avraham, and Shlomo Havlin. Breakdown of the internet under intentional attack. Phys. Rev. Lett., 86:3682, 2001.
[7] Duncan S. Callaway, M. E. J. Newman, Steven H. Strogatz, and Duncan J. Watts. Network robustness and fragility: Percolation on random graphs. Phys. Rev. Lett., 85:5468, 2000.

[8] Petter Holme and Beom Jun Kim. Attack vulnerability of complex networks. Phys. Rev. E, 65:056109, 2002.

[9] Lazaros K. Gallos, Reuven Cohen, Panos Argyrakis, Armin Bunde, and Shlomo Havlin. Stability and topology of scale-free networks under attack and defense strategies. Phys. Rev. Lett., 94:188701, 2005.

[10] Adilson E. Motter. Cascade control and defence in complex networks. Phys. Rev. Lett., 93(9):098701, 2004.

[11] L. Zhao, K. Park, and Y.-C. Lai. Attack vulnerability of scale-free networks due to cascading breakdown. Phys. Rev. E, 70:035101(R), 2004.

[12] Luciano da Fontoura Costa. Reinforcing the resilience of 
complex networks. Phys. Rev. E, 69:066127, 2004.

[13] T. Tanizawa, G. Paul, R. Cohen, S. Havlin, and H. E. Stanley. Optimization of network robustness to waves of targeted and random attacks. Phys. Rev. E, 71:047101, 2005.

[14] Vito Latora and Massimo Marchiori. Vulnerability and protection of infrastructure networks. Phys. Rev. E, 71:015103(R), 2005.

[15] Mirko Schäfer, Jan Scholz, and Martin Greiner. Proactive robustness control of heterogeneously loaded networks. Phys. Rev. Lett., 96:108701, 2006.

[16] Luca DallAsta, Alain Barrat, Marc Barthélemy, and Alessandro Vespignani. Vulnerability of weighted networks. physics/0603163 2006.

[17] M. Kurant and P. Thiran. Trainspotting: Extraction and analysis of traffic and topologies of transportation networks. physics/0510151, accepted for publication in Phys. Rev. E, 2005.

[18] http://www.netdimes.org

[19] http://www.caida.org/

[20] Daniel Stutzbach, Reza Rejaie, and Subhabrata Sen. Characterizing unstructured overlay topologies in modern p2p file-sharing systems. Proc. of IMC'05, 2005.

[21] Hagmann P., Kurant M., Gigandet X., Thiran P., Wedeen V., Meuli R., and Thiran J.P. Mapping brain networks of structural connectivity with MRI tractography. Manuscript in preparation, 2006.

[22] James F. Kurose and Keith W. Ross. Computer Networking. Addison Wesley, 2003.

[23] L. Gao. On inferring autonomous system relationships in the internet. IEEE/ACM Transactions on Networking, 9(6):733-745, 2001.

[24] M. E. J. Newman and M. Girvan. Finding and evaluating community structure in networks. Phys. Rev. E, 69:026113, 2004.

[25] This phenomenon is similar in spirit to the clustering algorithm proposed by Newman [24]. There, at every iteration, the edge with the highest betweenness is deleted. (The betweenness of a vertex or an edge is the fraction of shortest paths between all pairs of vertices in a network, that pass through it.) This results in physical graph partitions that correspond to its clusters (or 'communities'). It can be viewed as a special case of our attack, i.e., assuming the logical topology a fully connected unweighted graph. However, as the real-life traffic patterns are much more heterogenous [1], the attack under full rerouting produces partitions that correspond to the high traffic cut-sets in the physical graph, rather than to communities. 